First publ. in: Surface Science 113 (1982), 1-3, pp. 405-411

\title{
MACROSCOPIC ELECTRON DIMPLES ON THE SURFACE OF LIQUID HELIUM
}

\author{
P. LEIDERER, W. EBNER and V.B. SHIKIN * \\ Fachbereich Physik der Techmischen Universität München, D-8046 Ganching. Fed. Rep. of Germam!
}

We have investigated the sclf-trapping of electrons in macroscopic dimples on the surface of liquid ${ }^{4} \mathrm{Hc}$. The existence of individual multielectron dimples is demonstrated.

\section{Introduction}

Liquid helium, due to its unique properties, provides a nearly ideal flat substrate for the investigation of two-dimensional electron systems [1]. The coupling between the charges and excitations of this substrate in general is so weak that often it can be neglected. For some recent measurements, however, like experiments on the microscopic two-dimensional electron crystal [2] and the softening of ripplons [3] the interaction between the Coulomb system and the liquid surface is quite essential. As the most drastic effect the coupling leads to an electrohydrodynamic instability $[4,5]$ when the electric field perpendicular to the surface exceeds a critical value $E_{c}$, and thus limits the maximum attainable electron density on a homogeneously charged ${ }^{4} \mathrm{He}$ surface to $n \simeq 2 \times 10^{9} \mathrm{~cm}^{-2}$.

The development of the surface instability is a result of the softening of surface waves in the presence of the Coulomb system. The softening is most pronounced for modes with a wave vector $q=q_{\mathrm{c}}=1 / a$ (where $a$ is the capillary length of the liquid) whose frequency vanishes for $E \rightarrow E_{\mathrm{c}}$. As a result the surface deforms spontaneously as the critical field is reached, and a wave-like pattern with $q=q_{\mathrm{c}}$ appears, which then breaks up further into a periodic array of macroscopic dimples (see fig. l). Simultaneously the originally homogeneous charge distribution is destroyed because the electrons aggregate in the dimples. Investigations of the properties of this dimple crystal close to the transition, i.e. for fields $(E-E)_{\mathrm{c}} / E_{\mathrm{c}} \ll 1$, have shown a similarity to first order phase transitions $[5,6]$. Here we present results for dimples at

* Permanent address: Institule of Solid State Physics. Academy of Sciences of the USSR, Chernogolovka, Moscow District, USSR.

Konstanzer Online-Publikations-System (KOPS)

URL: http://www.ub.uni-konstanz.de/kops/volltexte/2007/2924/

URN: http://nbn-resolving.de/urn:nbn:de:bsz:352-opus-29243 

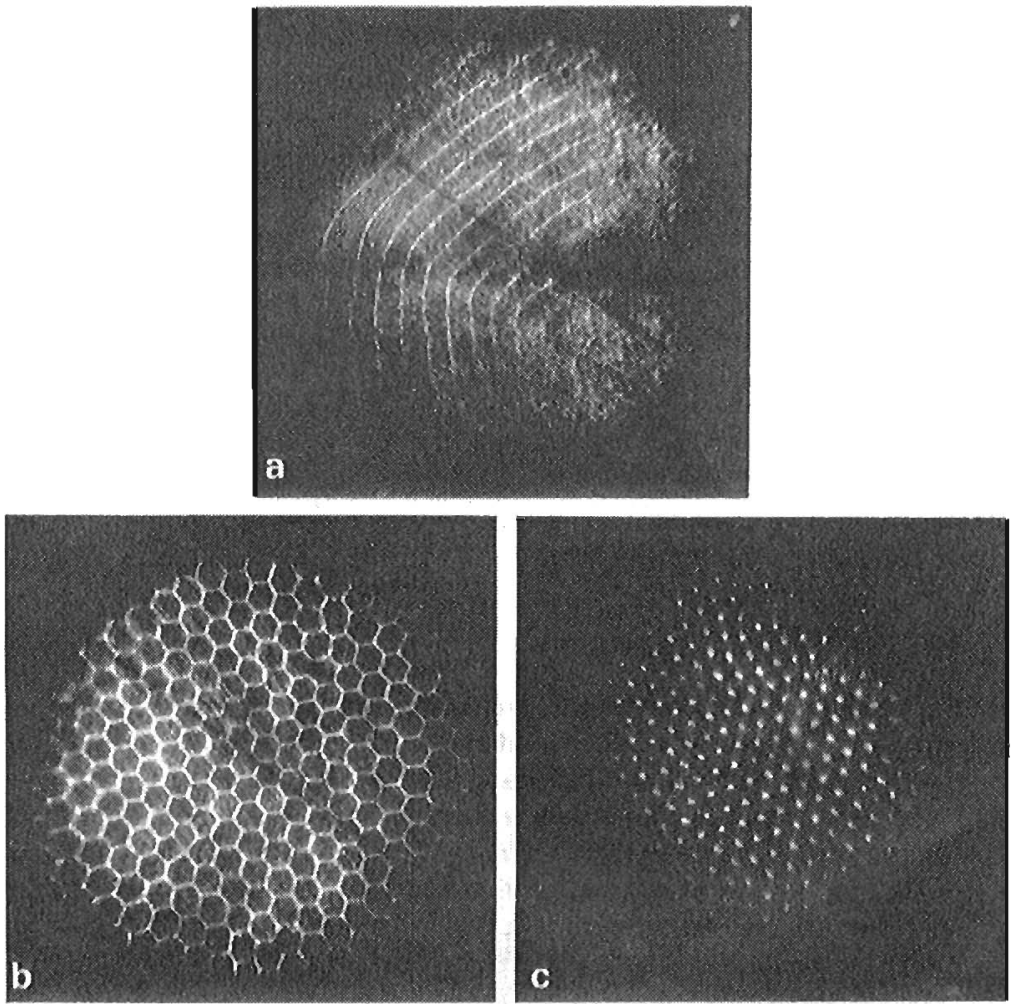

Fig. 1. Formation of a dimple lattice on a ${ }^{4} \mathrm{Hc}$ surface $(T=3.5 \mathrm{~K})$ charged with electrons from above. The pictures show the surface deformation approximately $2 \mathrm{~s}(\mathrm{a})$ and $6 \mathrm{~s}((\mathrm{~b})$ and (c)) after the field had been increased to $E_{c}$. The image plane in (a) and (b) was choscn such that convex deformations of the surface, corresponding to local maxima, appear bright; in (c) bright areas correspond to local minima (i.e. the center of the dimples). The distance betwcen adjacent rows of dimples is close to the wavelength $2 \pi a$ of the soft ripplon, $0.24 \mathrm{~cm}$ in this casc.

fields well above $E_{c}$, and in particular deal with individual multielectron dimples.

\section{Theoretical}

The instability of a charged liquid helium surface in high electric fields has first been considered by Gorkov and Chernikova [7], and in more detail by Ikezi [8], who for $E \geqslant E_{\mathrm{c}}$ obtained periodic deformations by including nonlin- 
ear terms in the equation of motion of the charged surface.

Extending these theories to fields distinctly larger than $E_{c}$, where the surface deformations become large, would require taking into account terms of still higher order. We have here chosen a different approach, considering the stability of a single macroscopic dimple. Both the deformation of the surface and the charge distribution in the dimples were determined by means of a self-consistent numerical calculation [9]. Starting from some fixed initial configuration of electrons, $n_{0}(r)$, and an external field $E$, the local depression $z(r)$ of the liquid surface was obtained by minimizing the total energy of the dimple, which consists of contributions from gravity, surface tension, and electrostatic pressure:

$$
W_{\text {tot }} \approx \int\left\{\frac{1}{2} \rho g z^{2}+\sigma\left[\left(1+z^{\prime 2}\right)^{1 / 2}-1\right]+z n_{0}(r) \mathrm{e} E+w_{\text {coulomb }}\right\} 2 \pi r \mathrm{~d} r .
$$

Here $\rho$ is the density of the liquid, $g$ is the acceleration of gravity and $\sigma$ is the surface tension. (The density of the vapor is neglected here, as well as the deviation of the dielectric constant of $\mathrm{He}$ from 1.) Notice that the energy $w_{\text {Coulomb }}$ due to the electrostatic repulsion energy of the electrons in the dimple is constant because at this stage $n_{0}(r)$ is fixed. From the surface deformation found in this way a new electron configuration $n_{1}(r)$ was determined - using the condition of constant electrostatic potential within the charge distribution and keeping the total charge of the dimple

$Q=e \int n(r) 2 \pi r \mathrm{~d} r$

fixed - and then the procedure was repeated.

The stability of such a localized charge distribution on the surface of liquid helium depends on the applied electric field and the number $N$ of electrons in the dimple:

(i) At high fields and large $N$ the charges become more concentrated at the

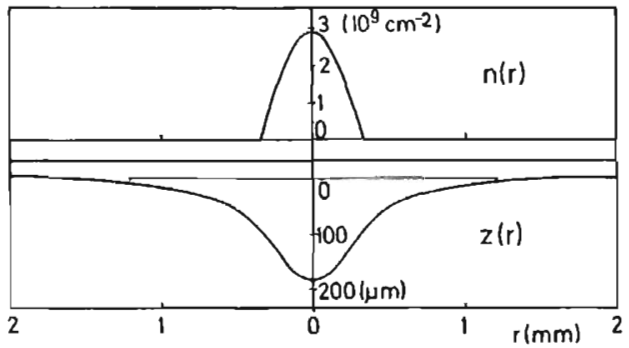

Fig. 2. Calculated electron density $n(r)$ and surface profile $z(r)$ of a dimple containing $5 \times 10^{6}$ electrons in an electric ficld $E=3400 \mathrm{~V} / \mathrm{cm}$. The helium temperature is $2.5 \mathrm{~K}$, the corresponding critical ficld $E_{c}=\left(16 \pi^{2} g \nu \sigma\right)^{1 / 4}$ is $2600 \mathrm{~V} / \mathrm{cm}$ [5]. 
center of the dimple in each iteration step, and the dimple depth increases indefinitely, leading to a breakthrough of the charges as expected.

(ii) At low fields the charges spread out more and more, indicating the formation of a homogeneously distributed layer of electrons at the surface.

(iii) For an intermediate range of fields above $E_{\mathrm{c}}$, a stable configuration can be obtained after several iterations if the number of electrons in the dimple is below a limit $N_{\mathrm{c}}(E)$. An example for the surface profile and charge density of such an isolated dimple is given in fig. 2. (Note that $n(r)$ in the center of the dimple is larger than the maximum attainable electron density of a homogeneously charged surface, which is $n_{\max }=2.1 \times 10^{9} / \mathrm{cm}^{2}$ at that temperature [5].)

As fig. 2 shows, dimples can exist as isolated entities, with the electrons being completely confined to the center of the dimple. Since, due to this self-trapping of the electrons, the charge in each dimple is fixed, the interaction between individual dimples is dominated by Coulomb repulsion. An arrangement of such "deep" dimples at the surface of liquid helium can therefore be considered as a macroscopic two-dimensional Coulomb system; in contrast, for the "shallow" dimple lattice close to $E_{c}$, as calculated by Ikezi [8], the electron density at the surface nowhere vanishes, but is only modulated and might thus be regarded as a charge density wave.

\section{Experiments}

The existence of individual dimples was confirmed by measurements in a sample cell consisting of two horizontal capacitor plates (spacing $1.5 \mathrm{~cm}$ ) and a cylindrical wall (diameter $8 \mathrm{~cm}$ ). The lower capacitor plate was a gold mirror, the upper was a glass plate covered with a transparent conductive coating which allowed observation of the helium surface from above. Starting with an uncharged ${ }^{4} \mathrm{He}$ surface and an applied electric field $E>E_{c}$, a pulse of electrons was generated by momentarily heating a small filament located close to the
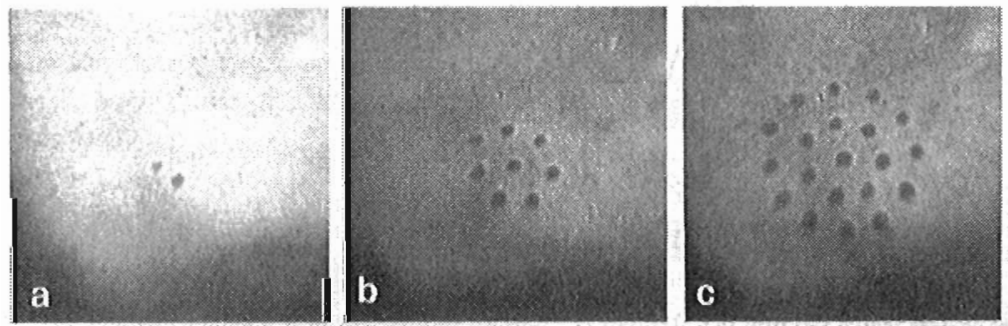

Fig. 3. Configurations of (a) 2, (b) 8 and (c) 20 dimples (appearing as dark spots) in an external potential of cylindrical symmetry. 

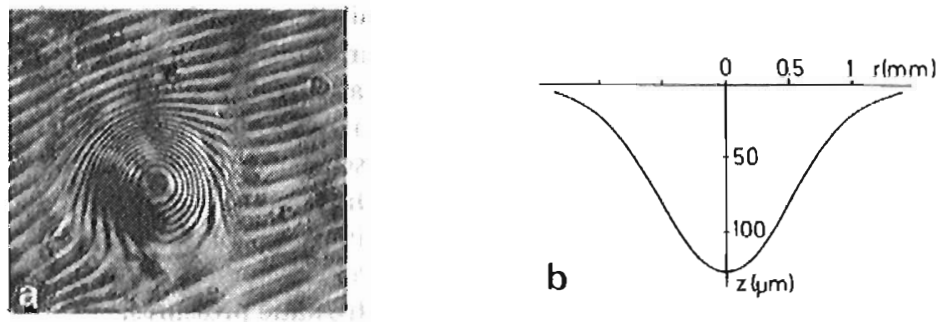

Fig. 4 (a) Interference pattern of a macroscopic dimple $\left(N \sim 5 \times 10^{6}\right.$ electrons, $T=2.5 \mathrm{~K} . E=2.9 \times$ $10^{3} \mathrm{~V} / \mathrm{cm}$ ). The fringe pattern is asymmetric because the two interferometer plates werc slightly tilted. Consecutive rringes correspond to a difference in surface height of $10.9 \mu \mathrm{m}$. (b) The surface profile derived from (a).

circumference of the cell. The emitted electrons were pulled against the surface and then formed a dimple with a charge $Q$ being determined by the emission of the source. Since a negative voltage, applied to the wall of the sample cell, gives rise to cylindrically symmetric potential, the dimple after being generated drifts to the potential minimum at the center of the cell. As more dimples are added they arrange themselves leading to configurations illustrated in fig. 3. Upon increasing the number of constituents further, a hexagonal lattice develops gradually which is also observed when the dimple state is produced from a homogeneous charge distribution by raising the electric field beyond the instability threshold [5] (see also fig. 1).

The deformation of the liquid surface near a dimple can be determined with high resolution by analyzing the interference pattern obtained when coherent light reflected from the top and from the bottom capacitor plate is superimposed. Fig. 4 shows an example of such an interference pattern and the corresponding dimple profile. Although the number of electrons in this dimple
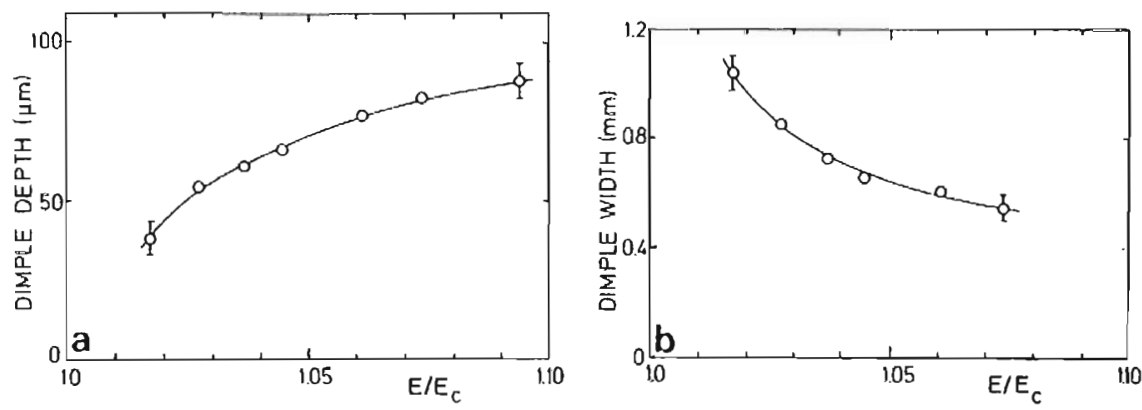

Fig. 5. Experimental depth and width (full width at half minimum) of a dimple with constant charge vs. the reduced electric field $E / E_{\mathrm{c}}(T=4.2 \mathrm{~K})$. The lines are only guides to the eyc. 
was not known very accurately and hence a detailed comparison with the numerical calculations is not yet possible, the similarity between the shapes of calculated and experimental dimple profiles (figs. 2 and 4 ) is obvious.

Once a dimple is formed, its profile varies as the electrical field is raised further: The depth increases, and its width shrinks, so that the whole structure becomes sharper (see fig. 5). Consequently at higher fields the electrons become more concentrated at the dimple center. When the dimple profile is known, the charge density $n(r=0)$ at the center can be derived from the condition of equilibrium between gravitational, surface, and electrostatic pressures:

$n(r=0) \cdot e E \simeq 2 \sigma / R+\rho g h$.

Here $R$ is the radius of surface curvature at the dimple center and $h$ is the depth of the dimple. Experimental results for the dimple profile confirm our conclusion from the numerical calculation that at the dimple center an electron density higher than $n_{\max }$ (of the homogeneously charged surface) can be reached.

The dimples discussed so far always were formed by electrons pulled against the helium surface from above. With a field ionization tip located below the helium surfacc, on the other hand, it is possible to generate positive ions in the bulk liquid, which, when pulled upwards, form a two-dimensional sheet of positive charges just below the helium surface. The electrohydrodynamic instability and the dimple lattice can be observed under these conditions as well; in particular, the critical field for dimple formation is the same. Both for purely positive and for purely negative dimple lattices the interaction between the dimples is essentially determined by Coulomb repulsion. How would a system behave with positively and negatively charged dimples being present at the helium surface simultaneously?

Although it is somewhat difficult to stabilize such a system experimentally [10], we have succeeded in producing a "dilute" aggregation of positive and negative dimples for a limited amount of time (a few minutes). Distances between dimples of the same sign in this case were large, but Coulomb attraction led to the formation of bound dimple pairs with opposite charge, or "dipoles", as sketched in fig. 6. Such complexes are stable because, in addition to the attractive potential, a repulsive potential due to the surface deformation and the external field exists, which prevents the two oppositely charged dimples from approaching too closely; a recombination of electrons and positive ions thus cannot take place, because, as already shown for individual

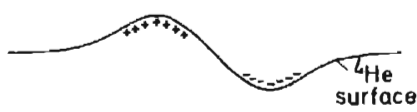

Fig. 6. Schematic profile as observed for a bound pair of dimples with opposite charge. 
dimples, each kind of charge carriers is trapped close to the center of its corresponding dimple. Hence the existence of bound dimple pairs is a further indication that dimples can be considered as macroscopic Coulomb particles with a fixed charge.

\section{Acknowledgements}

We gratefully acknowledge discussions with Professor $\mathrm{H}$. Kinder and Dr. M. Wanner, and contributions to the experiments by D. Savignac. This work was supported by the Deutsche Forschungsgemeinschaft.

\section{References}

[1] The most recent review on this subject is given by V.S. Edelman, Usp. Fiz. Nauk 130 (1980) 675 [Sovict l'hys.-Usp. 23 (1980) 227].

[2] C.C. Grimes and G. Adams, Phys. Rev. Letters 42 (1979) 795.

[3] M. Wanner and P. Leiderer, Phys. Rev. Letters 42 (1979) 315; P. Leiderer, Phys. Rev. B20 (1979) 451.

[4] M.S. Khaikin, J. Physique C.6 (1978) 1295.

[5] P. Leiderer and M. Wanner, Phys. Letters 73A (1979) 189 W. Ebner and P. Leiderer, Phys. Letters 80A (1980) 277.

(6) R.W. Gianetta and H. Ikezi, preprint.

[7] L.P. Gorkov and D.M. Chernikova, Pis'ma ZhETF 18 (1973) 119 (JETP Letters 18 (1973) 68); Dokl. Akad. Nauk SSSR (1976) 829 [Soviet Phys.-Dokl. 21 (1976) 328].

18) H. Ikezi. Phys. Rev. Letlers 42 (1979) 1688.

19] Other treatments of individual macroscopic dimples have been given by: V.B. Shikin and P. Leiderer, Pis'ma ZhETF 32 (1980) 439 [JETP Lelters 32 (1980) 416]: W.I. Melnikov and S.W. Meshkov. Pis'ma ZhETF 33 (1981) 222.

[10] In this case it is no longer possible to use adequate wall potentials to keep the dimples logether, because a potential chosen such that ncgative charges are confined will let positive charges escape to the wall, and vice versa. 